\title{
Clustering Techniques for Recognition of ADL Performed with Upper Limbs
}

\author{
Sergio Parra-Sánchez ${ }^{1}$, Juan Manuel Gómez-González ${ }^{2}$, A. Iraís Quintero-Ortega ${ }^{1}$, \\ Birzabith Mendoza-Novelo ${ }^{1}$, José Jorge Delgado-Garca ${ }^{1}$, Arturo Vega-González ${ }^{1}$ \\ ${ }^{1}$ Universidad de Guanajuato, División de Ciencias e Ingenierías, Guanajuato, Mexico \\ ${ }^{2}$ Universidad Nacional Autónoma de México, Facultad de Ingeniería, Mexico \\ spsoaz@gmail.com, avega@fisica.ugto.mx
}

\begin{abstract}
Activities of Daily Living (ADL) have a background of selfsufficiency and survival function. Upper limbs participate actively in many ADL; particularly, activities related to feeding, communication, and grooming. The performance of such activities is a parameter of independence. Various researchers have studied ADL in a free-living environment by using inertial sensors. However, functional-activity recognition with low recognition rate is a persistent result. This work proposes the use of well-known clustering techniques for $\mathrm{ADL}$ recognition by using as feeding signal the vertical trajectory of wrist relative to the shoulder.
\end{abstract}

Keywords: activities of daily living, clustering, recognition of ADL.

\section{Introduction}

The International Classification of Functioning and Disability (ICF) define functioning as the positive aspects of the interaction between an individual (with a health condition) and that individual's contextual factors (environmental and personal factors) [1]. Hence, the interaction is evaluated through the ADL. Thus, by recognizing ADL during an extended period, we can evaluate functioning.

When a person has a disability that limits his/her movement, it is necessary to obtain information about his/her functional state. In other words, there is a need to know what the person does, and how does he/she perform. Thus, measuring functional movements has become paramount for rehabilitation interventions [2]. Therefore, it is necessary to get information about ADL performed by the person in a real-life scenario. For upperlimb movements, the most common sensors are accelerometers and gyroscopes [2-5]. However, the recognition of activities by using acceleration signals or any other device or combination of them have not shown acceptable results in an extensive set of activities.

The goal of this work was the evaluation of the Upper-limb functional-activity recognition based on clustering techniques. The target activities include eating, drinking, talking by phone, write on a computer, brushing teeth and combing hair. The input signal for this purpose was the vertical displacement of the wrist relative to the shoulder. 


\subsection{Clustering}

Clustering is one of the most used techniques in the recognition of Activities of Daily Living [2-11]. Clustering analysis is the formal study of algorithms and methods to group objects according to measurements, perceived attributes, intrinsic features or likelihoods [12]. This technique consists of patterns classification (observations, signals, vectors) into groups (clusters). Every cluster shares common features that make it similar among their objects, and at the same time, these features make different from objects of another cluster or group.

\section{Methods}

\subsection{Participants}

Eight participants ( 7 men and one woman) between 23 and 42 ages were recruited. The exclusion criterion was upper-limb motor impairment. The measurement protocol was according to the Mexican norm NOM-012-SSA3-2012 and the Helsinki declaration of the World Medical Association (WMA). All participants signed a consent-informed letter, and the research protocol was approved by the institution's ethical committee (CIBIUG-EX01-2018).

\subsection{Measurement Protocol}

Participants wore an electro-hydraulic sensor able to measure the vertical component between two points. This device was located at the shoulder of the participants to measure the vertical component of the distance between wrist and shoulder during the execution of a set of activities. The participants were video-recorded while they performed a set of ADL.

\subsection{Data Analysis and Clustering Recognition}

Displacement's signals were filtered using a low-pass filter with $10 \mathrm{~Hz}$ cut-off frequency, and then were extracted the following set of features: maximum normalized vertical position, minimum normalized vertical position, standard deviation of vertical position, maximum vertical velocity, minimum vertical velocity, maximum vertical acceleration, minimum vertical acceleration, average of acceleration, Spearman correlation between arms, kurtosis of position, three significant frequencies. Then, these features were reduced to five features by using Principal Components Analysis (PCA). PCA is a dimensional reduction technique based on axes rotation, new axes obtained are related to maximized variance. In this new space, the following clustering techniques were used to analyse data space: k-means, DBSCAN, and spectral clustering [13]. In this technique, the adjacency matrix was built by using a Gaussian similarity. A brief description of every one of the algorithms used in this research is presented in the following paragraphs. 
k-means: The algorithm considers a data set $\mathbf{X}$, and a set of clusters $\mathrm{C}=\left\{\mathrm{C}_{1}, \ldots, \mathrm{Ck}\right\}$. Every cluster $C_{k}$ is related to a point called centroid $\mathbf{x}_{\mathbf{C k}}$. Each observation or data point relates to the nearest cluster by a similarity function, such as the Euclidian distance. Thus by minimizing the sum of the distances of every point belonging to cluster $C_{k}$, it is possible to partition the data set.

DBSCAN: DBSCAN (Density-Based Spatial Clustering of Applications with Noise) is an algorithm to discover arbitrarily shaped clusters. In this algorithm, a cluster is formed by dense regions (a portion of data space where the number of points is more significant than a criterion selected by the user). With this method exist the possibility to detect noise, being these, isolated points.

Spectral clustering: Spectral clustering is a graph-based algorithm, which often outperforms the traditional approaches [13]. In this algorithm, the dataspace is transformed to a graph (a set of dots joined among them), to do this; it is necessary a similarity measure, then all pairs of data are compared among them. Thus, similar data are nearer than different. By using the information about the structure of the graph, a matrix is built; finally, the eigenvectors of this matrix feed the k-means algorithm.

\subsection{Clustering Evaluation}

Relative evaluation of clustering methods was done by using internal and external indexes. The coefficients used are presented in the table 1. Indexes shown refers to the parameters defined in the following paragraph.

Consider $C=\left\{\mathrm{C}_{1}, \ldots, \mathrm{C}_{\mathrm{k}}\right\}$, a clustering structure of a data set $\mathbf{X}$ and $P=\left\{\mathrm{P}_{1}, \ldots, \mathrm{P}_{\mathrm{s}}\right\}$ is a defined partition of the data given by the user, in this sense, the partition reflects the intuition about data of the user. Then, refer to a pair of points $\left(\mathbf{x}_{\mathrm{v}}, \mathbf{x}_{\mathrm{u}}\right)$ from the dataset using the following terms:

- SS: if both points belong to the same cluster of the clustering structure $C$ and to the same group of partition $P$.

- SD: if points belong to the same cluster of $C$ and to different groups of $P$.

- DS: if points belong to distinct clusters of $C$ and to the same group of $P$.

- DD: if both points belong to different clusters of $C$ and to distinct groups of $P$.

Now, let a, b, c and d the numbers of pairs in SS, SD, DS and DD respectively, then $a+b+c+d=M$. Which it is the number of all pairs formed with the data set. Let $a, b$, $c$ and $d$ be the quantity of pairs belonging to class: SS, SD, DS, DD.

In addition, a clustering validation was done using a random test based on Monte Carlo method [14], this method consists in the creation of artificial data (pseudo randomly) in the same dataspace, then applying the same algorithms and relative indexes is obtained a new result. This procedure was repeated 1000 times and as a result, a histogram was built; with this, it is possible to indicate differences in clustering results of our data and a data with a pseudo random structure. Then, the method is used to corroborate the non-random structure of data. 


\section{Results and Discussion}

Relative evaluation showed that the best recognition can be obtained using three clusters and becomes worst while this number increases.

Table 1 shows results of the relative evaluation index for three clusters only. In this table, the spectral clustering technique showed the poorest score, indicating that the kind of graph used does not reflect the cluster structure adequately. On the other hand, DBSCAN showed that data space is density-in-homogeneous due to the small results. On the other hand, the table 3 shows the percentage of recognition for each algorithm. Cells of the same colour per row belong to the same cluster.

Table 2 shows that the best clustering used was the k-means with a recognition score up to $90 \%$ for three clusters.

In addition, the Table 3 shows the recognition score for the different functional tasks. Finally, the random test evaluation indicates a non-random structure of data for all algorithms with a confidence interval up to $95 \%$. This result agrees with those shown in table 2.

The Random test indicates a non-random structure of data for all algorithms and most of the coefficients used a confidence interval of $95 \%$. The best technique was $\mathrm{k}-$ means (using 3 clusters) with recognition score up to $90 \%$. Spectral clustering techniques showed a poor score, this indicates the graph does not reflect the cluster structure adequately. Furthermore, DBSCAN showed that data space is densityinhomogeneous.

These results suggest that clustering can be used to perform a recognition of ADL and could be used in an initial step as pre-processing, in more sophisticated recognition algorithms.

Table 1. Different coefficients used to evaluate clustering.

\begin{tabular}{|c|c|c|}
\hline Index & Expression & Reading \\
\hline Fowlkes and Mallows ind & $F M=\sqrt{\left(\frac{\mathrm{a}}{\mathrm{a}+\mathrm{b}}\right)\left(\frac{\mathrm{a}}{\mathrm{a}+\mathrm{c}}\right)}$ & Bigger the better \\
\hline Jaccard coefficient & & Bigger the better \\
\hline Silhouette & $\begin{array}{l}S= \begin{cases}1-\frac{A}{B} & \text { if } A \leq B \\
\frac{B}{A}-1 & \text { if } A \geq B\end{cases} \\
A=\frac{1}{N_{j}} \sum_{x_{j} \in C_{i}}\left\|\boldsymbol{x}_{i}-\boldsymbol{x}_{j}\right\|^{2} \\
B=\min \left(\frac{1}{N_{-i}} \sum_{x_{j} \notin C_{i}}\left\|\boldsymbol{x}_{j}-\boldsymbol{x}_{i}\right\|^{2}\right)\end{array}$ & Bigger the better \\
\hline $\begin{array}{l}\text { Rand statistic } \\
\text { Cohesion parameter } \\
\text { Separation measurement }\end{array}$ & $\begin{array}{l}R=\frac{\mathbf{a}+\mathbf{d}}{\mathbf{a}+\mathbf{b}+\mathbf{c}+\mathbf{d}} \\
S S E=\sum_{C_{j}=1}^{k} \sum_{i \in=C_{j}}\left\|\boldsymbol{x}_{i}-\boldsymbol{x}_{C_{j}}\right\| \\
S S B=\sum_{j} N_{j}\left\|x_{C_{j}}-\boldsymbol{\mu}\right\|^{2}\end{array}$ & $\begin{array}{l}\text { Bigger the better } \\
\text { Smaller the better } \\
\text { Bigger the better }\end{array}$ \\
\hline
\end{tabular}

Table 2. Best scores for everyone algorithm analyzed using three clusters. NA indicates that the index cannot be applied due to the incompatibility between clustering algorithm and evaluation index.

\begin{tabular}{|c|c|c|c|c|c|c|}
\hline Algorithm & $\begin{array}{c}\text { Rand } \\
\text { Statistic }\end{array}$ & $\begin{array}{c}\text { Fowlkes \& } \\
\text { Mallows }\end{array}$ & Jaccard & SSB & SSE & Silhouette \\
\hline K-means & 1.80 & 0.76 & 0.86 & 0.21 & 6.17 & 0.51 \\
\hline DBSCAN & 1.6 & 0.7 & 0.55 & NA & NA & NA \\
\hline Uspectral & 1.19 & 0.36 & 0.59 & NA & NA & NA \\
\hline
\end{tabular}




\section{Conclusions}

The K-means algorithm can group activities that belong to the same task; making easier the recognition process. Our results are promissory about the improvement of recognition rate in more sophisticated algorithms using the clustering as a preprocessing step. The clustering techniques can recognize functional activity in a categorized form.

Table 3. Recognition scores obtained for three clusters. Percentages of recognition were calculated using the known data partition. In table USC, NSCa and NSCb represents the algorithms: no-normalized spectral clustering, Normalized spectral clustering according to Shi and Malik, and Normalized spectral clustering according to Jordan, $\mathrm{Ng}$, and Weiss. The cells with the same font color are in the same cluster, divided cells have a division of data between two clusters.

\begin{tabular}{|c|c|c|c|c|c|c|}
\hline Algorithm & Eating & Drinking & $\begin{array}{c}\text { Talking by } \\
\text { phone }\end{array}$ & $\begin{array}{c}\text { Writing on } \\
\text { computer }\end{array}$ & $\begin{array}{c}\text { Combing } \\
\text { hair }\end{array}$ & $\begin{array}{c}\text { Brushing } \\
\text { teeth }\end{array}$ \\
\hline K-means & $93.7 \%$ & $100 \%$ & $80.6 \%$ & $100 \%$ & $69.8 \%$ & $89.6 \%$ \\
\hline DBSCAN & $87.93 \%$ & $92.65 \%$ & $83.33 \%$ & $91.67 \%$ & $96.23 \%$ & $93.62 \%$ \\
\hline USC & $81 \%$ & $54 \%$ & $62 \%$ & $88 \%$ & $77 \%$ & $51 \%$ \\
\hline NSCb & $59.46 \%$ & $85.24 \%$ & $79.17 \%$ & $86.11 \%$ & $49.06 \%$ & $44.68 \%$ \\
\hline NSCa & $61.26 \%$ & $85.29 \%$ & $77.08 \%$ & $80.56 \%$ & $40.06 \%$ & $46.81 \%$ \\
\hline
\end{tabular}

Acknowledgments. We thank the support by DAIP-CIIC 319/2018.

\section{References}

1. World Health Organization: ICF: International classification of functioning, disability and health. World Health Organization Geneva (2001)

2. Uswatte, G., Miltner, W.H.R., Foo, B., Varma, M., Taub, S.M.E.: Ambulatory monitoring of arm movement using accelerometry: An objective measure of upper-extremity rehabilitation in persons with chronic stroke. Stroke journal of the American Heart Association 31, pp. 662-667 (2005)

3. Biswas, D., Cranny, A., Gupta, N., Maharatna, K., Achner, J., Klemke, J., Jöbges, M., Ortmann, S.: Recognizing upper limb movements with wrist worn inertial sensors using k-means clustering classification. Hum. Mov. Sci. 40, pp. 59-76 (2015)

4. Avgerinakis, K., Briassouli, A., Kompatsiaris, I.: Activity detection and recognition of daily living events. In: Proceedings of the 1st ACM International Workshop on Multimedia Indexing and Information Retrieval for Healthcare. MIIRH'13, New York, NY, USA, ACM, pp. 3-10 (2013)

5. Zillmer, R.: Measurement of toothbrushing behaviour in a natural environment. Pers. Ubiquit. Comput. 17, pp. 29-33 (2011)

6. Prabowo, O.M., Mutijarsa, K., Supangkat, S.H.: Missing data handling using machine learning for human activity recognition on mobile device. In: 2016 International Conference on ICT for Smart Society (ICISS), pp. 59-62 (2016)

7. Chetty, G., White, M.: Body sensor networks for human activity recognition. In: 2016 3rd International Conference on Signal Processing and Integrated Networks (SPIN), pp. 660665 (2016)

8. Batchuluun, G., Kim, J.H., Hong, H.G., Kang, J.K., Park, K.R.: Fuzzy system based human behavior recognition by combining behavior prediction and recognition. Expert Systems with Applications 81, pp. 108-133 (2017) 
9. Biswas, D., Cranny, A., Gupta, N., Maharatna, K., Ortmann, S.: Recognition of elementary upper limb movements in an activity of daily living using data from wrist mounted accelerometers. In: Proceedings-2014 IEEE International Conference on Healthcare Informatics, ICHI 2014, pp. 232-237 (2014)

10. Yazdansepas, D., Niazi, A.H., Gay, J.L., Maier, F.W., Ramaswamy, L., Rasheed, K., Buman, M.P.: A multi-featured approach for wearable sensor-based human activity recognition. In: 2016 IEEE International Conference on Healthcare Informatics (ICHI), pp. 423-431 (2016)

11. Serrano, J.I., Lambrecht, S., del Castillo, M.D., Romero, J.P., Benito-Len, J., Rocon, E.: Identification of activities of daily living in tremorous patients using inertial sensors. Expert Systems with Applications 83, pp. 40-48 (2017)

12. Jain, A.K.: Data clustering: 50 years beyond k-means. Pattern Recognition Letters 31, pp. 651-666 (2010)

13. Planck, M., Luxburg, U.V.: A Tutorial on Spectral Clustering. Stat. Comput. 17, pp. 395$416(2006)$

14. Sergios, T., Konstantinos, K.: Pattern Recognition. Academic Press, Elsevier (2009) 